what the general tendency is as long as the object carries out this tendency. The essential thing is the fulfillment of a tendency of whatever sort for its own sake without involving any purpose. The æsthetic consciousness is, then, a state aroused by the objective fulfillment of a tendency regarded without reference to any ulterior end, and the function of the elements of the beautiful object is to suggest such tendency and at the same time to fulfill it.

\title{
A New Perimeter.
}

BY JAMES E. LOUGH.

Indirect vision is one of several problems of sight now under investigation in the Harvard Psychological Laboratory. For the purposes of this study the ordinary perimeter and campimeter have proven themselves almost useless. In these instruments the eye looks at a stationary point, whilst the stimulating object changes its place on the graduated arc. This change of place alters the objective lllumination, etc., of the object, so that the effect of fine changes in its intensity, size, etc., cannot be accurately studied. This difficulty has been overcome in the instrument here described by reversing the usual order of things and making the fixation point movable while the stimulus is the stationary part of the apparatus. By this arrangment the operator is given absolute control over the variations of the stimulus.

A description of this instrument is published now before any exact results can be reported, in the hope that it may prove helpful to others engaged in this same line of investigation.

Figure I shows the ground plan, a semi-cylinder of blackened brass. A, $30 \mathrm{~cm}$. high, with a radius of $30 \mathrm{~cm}$., is supported by a base board $60 \mathrm{~cm} . \times 40 \mathrm{~cm}$. and by back and side boards $B$ and $F$. In the middle of $A$ and extending through $B$ is a window, $W, 10 \mathrm{~cm} . x$ ro $\mathrm{cm}$. This opening may be filled 
whenever one of the holes in the circle coincides with the slit $O S$. The rotation of the circle about $X$ will vary the distance between these two points. This distance can be easily read to $\frac{1}{6} \mathrm{~mm}$. ( $\left.2^{\prime}\right)$ upon the scale $P$. The intensity of the stimulating light is easily regulated by the distance of the lamp, while the quality of the light may be varied by the use of gelatine sheets.

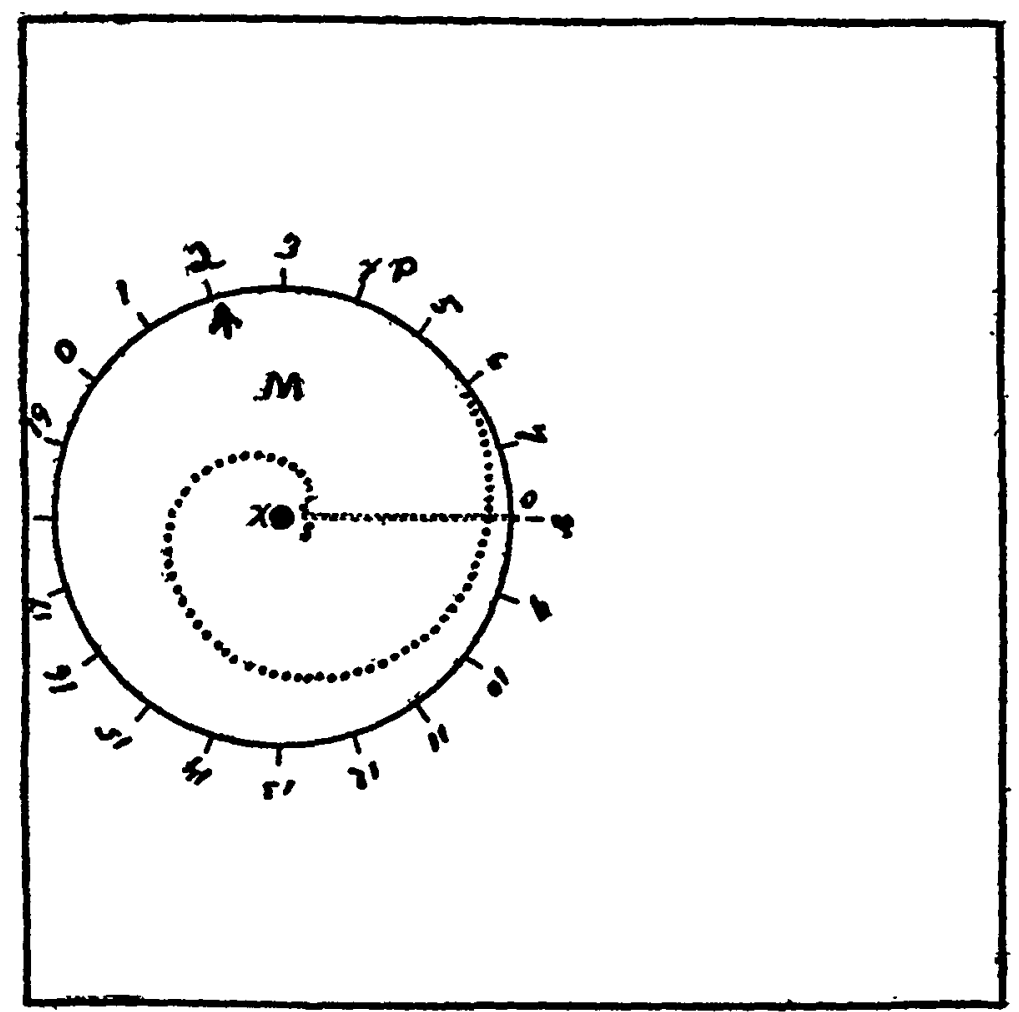

FIG. 2.

By this apparatus, therefore, the vertical and horizontal distance at which two points of light stimulating the retina appear as one (the retinal unit) may be obtained for all portions of the retina. And the influence of the intensity and the quality of light upon the retinal units may be determined.

Another shutter may be placed in the window $W$, having an opening at the center, the size of which is controlled by an iris 
diaphragm. By means of a lamp and gelatine sheets a light stimulus of any quality, intensity or size may be made to excite any portion of the retina. Such experiments quickly demonstrate that the 'color fields' depend entirely upon the size and intensity of the stimulating color.

This instrument facilitates the study of two points of different color within one retinal unit; of the threshold for colors; of the perception of differences in quality, in intensity and in position for all portions of the Iretina. Reports of these and of other investigations will be published as they are completed. 